\title{
3D analysis, modeling and simulation of transport processes in compressed fibrous microstructures, using the Lattice Boltzmann method
}

\author{
Dieter Froning $^{\mathrm{a}}$, Jan Brinkmann ${ }^{\mathrm{a}}$, Uwe Reimer ${ }^{\mathrm{a}}$, Volker Schmidt ${ }^{\mathrm{b}}$, Werner Lehnert ${ }^{\mathrm{a}, \mathrm{c}}$, Detlef Stolten ${ }^{\mathrm{a}, \mathrm{d}}$ \\ ${ }^{a}$ Forschungszentrum Jülich GmbH, Institute of Energy and Climate Research, IEK-3: Electrochemical Process Engineering, D-52425 Jülich, \\ Germany \\ ${ }^{b}$ Institute of Stochastics, Ulm University, Germany \\ ${ }^{c}$ Modeling in Electrochemical Process Engineering, RWTH Aachen University, Germany \\ ${ }^{d}$ Chair for Fuel Cells, RWTH Aachen University, Germany
}

\begin{abstract}
In this paper we combine a stochastic 3D microstructure model of a fiber based gas diffusion layer of polymer electrolyte fuel cells with a Lattice Boltzmann model for fluid transport. We focus on a simple approach of compressing the planar oriented virtual geometry of paper-type gas diffusion layer from Toray. Material parameters - permeability and tortuosity - are calculated from simulation of one phase, one component gas flow in stochastic geometries. We analyze the statistical spread of simulation results on ensembles of the virtual geometry, both uncompressed and compressed. The influence of the compression is discussed with regard to the Kozeny-Carman equation. The effective transport properties calculated from transport simulations in compressed gas diffusion layers agree well with a trend based on the Kozeny-Carman equation.
\end{abstract}

Keywords: paper-type GDL, compression, stochastic modeling, Lattice Boltzmann, Kozeny-Carman

\section{Introduction}

Because of their high efficiency and low emissions polymer electrolyte fuel cells (PEFC) are promising devices for electrical power generation. The major components of a typical PEFC are the membrane electrode assembly (MEA), the flow fields, and the gas diffusion layer (GDL) [1, 2]. The electrochemical conversion of hydrogen takes place at the electrodes with their typical pore sizes of about $100 \mathrm{~nm}$. The flow fields at the anode and cathode side are responsible for the transport of reacting gases to the membrane and to remove liquid and gaseous products. A typical cross section of channels in the flow fields on the anode and cathode side is $1 \mathrm{~mm}^{2}$. The GDLs are located between the flow field and the MEA on both the anode and the cathode side. Their function is to enable almost homogeneous mass transport between the channels and the MEA, not only under the channels but also under the ribs of the flow fields. Furthermore, the GDL has to provide electrical contact with the bipolar plates. GDLs can be produced as carbon paper, carbon cloth, and non-woven with typical fiber diameters of 5-10 $\mu \mathrm{m}$. The thickness is mostly

Email address: d.froning@fz-juelich.de (Dieter Froning) in the range of 100-300 $\mu \mathrm{m}[3,4]$. The microstructure of paper and non-woven is of random nature. The fibers may be glued together with binder, e.g., in case of carbon paper. If the GDL used in PEFCs operated below $100{ }^{\circ} \mathrm{C}$ at ambient pressure the hydrophobicity of the GDL is often accomplished by PTFE which is added to the GDL. The proper prediction of transport properties is challenging due to the complex geometry. When the GDL material is used in real fuel cell stacks, compression is applied by the flow field. This leads to different microstructures of the GDL in the regions under land and under channels. Lee et al. [5] studied the effects of compression and GDLs on the performance of a PEFC. The non-isotropic nature of the mass transport characteristics and their strong dependency from the compression was already observed by Hussaini and Wang [6]. Under compression the GDL structure is morphed under the ribs and material is intruded into the channels [7]. This effect is $43-125 \%$ higher for carbon cloth than for carbon paper. Poornesh et al. [8] found a relationship between the GDL's in-plane mechanical properties and membrane thinning. Parikh et al. [9] investigated the relationship between fuel cell efficiency and GDL materials by testing GDL samples from Freudenberg, 
SGL and Toray. They studied pore size distributions but also size, shape and orientation of the pores. Many groups concentrate their investigations on liquid water transport $[10,11]$ because this is a key functionality of the GDL when PEFCs are operated below $100^{\circ} \mathrm{C}$.

According to the different spatial scales of fuel cell components - $\mu \mathrm{m}$ scale for GDL structures and $\mathrm{mm}$ scale and above for flow fields - modeling approaches often concentrate on either of these scales. In modeling approaches of fuel cells or even stacks the GDL is often considered as a homogeneous medium with effective homogenized transport properties. With this approach Kvesić et al. were able to simulate the behavior of entire HT-PEFC stacks by embedding user defined functions for the electrochemical reactions into commercial CFD software [12, 13]. Also based on effective transport properties, the effect of different compression levels of the GDL was studied by Olesen et al. [14] by means of CFD. In their simulations the geometry was morphed under compression due to the calculated strain. The result is that inhomogeneous compression affects the local reaction rates in the active layer. Chippar et al. [15] observed similar results also with a CFD model. They considered the effect of compression in porous material parameters. Both - Olesen and Chippar - assumed homogeneous properties of the GDL material. For simulations of the GDL on $\mu \mathrm{m}$ scale we chose the LB method. With this method it is possible to incorporate complex spatial structures in the simulation domain. The features of the method range from single phase, single component flow $[16,17]$ to multi component $[18,19,20,21]$ and/or multi phase configurations [18, 22, 23, 24]. Sophisticated data structures and algorithms can be included $[25,26]$ as well as extended boundary conditions $[27,22,28]$. In this paper we present a LB based method to obtain effective material properties - permeability and tortuosity - of paper type GDLs. Mass transport focuses on single phase, single component flow of the anode of an HT-PEFC which is operated at $160{ }^{\circ} \mathrm{C}$. In this case the driving force for mass transport is forced convection because hydrogen is consumed at the electrode, which can be translated into a pressure gradient across the GDL.

Obtaining reliable transport properties requires knowledge both of the microstructure and of the transport processes - which was demonstrated by Hossain et al. [29]. They presented CFD simulations of a channel pair with focus on two phase species transport and reported in-plane and through-plane permeabilities of the GDL. A significant influence of the transport properties of the GDL on the cell voltage was observed.

Pore network models [30, 31, 32, 33, 34, 35] are widely used for simulating mass flow in GDLs, especially with emphasis of two phase flow. These models are very effective in order to capture the physics of mass transport in combination with evaporation and condensation. As a consequence of this approach the structure of the solid phase is either neglected or the pore network must be extracted from the real geometry by a separate modeling step - which is beyond the scope of this article.

Many groups use reconstructed GDL geometry in their modeling approaches which can be obtained from X-ray tomography [36, 37, 38]. Transport simulations on those microstructures can be preferably performed by the Lattice Boltzmann (LB) method [39]. Niu et al. [40] combined the multiple relaxation time (MRT) scheme of the LB method with the diffuse interface theory to simulate the multi phase transport in the GDL using reconstructed geometry of Toray paper. Ostadi, Rama et al. investigated in LB simulations on GDL structures which were obtained from nano-computed tomography. They characterized the structure according to geometric characteristics, anisotropy and effects of compression [41, 42, 21, 43, 44, 45, 46]. As in the macrohomogeneous approaches mentioned before also the simulations on the reconstructed microstructure show the evidence of the non-isotropic transport properties on the behavior of the mass transport on higher spatial scales.

To overcome the high expenses of tomography experiments virtual geometries are often generated by several methods. All the approaches have in common that the generated geometries show the same characteristics as the original structure. This can be done for generic fiber structures [47] and it can be simplified for straight fibers as they occur in paper-type GDL. The idea of representing fiber based material by randomly distributed cylinders was presented by Tomadakis and Sotirchos already in 1993 [48]. A commonly used stochastic model for fiber based GDLs was presented by Schladitz et al. [49]. In this model straight fibers of non-woven GDLs are generated by a 3D dilated Poisson line process. Its development was continued by Schulz et al. [50] and applied to characterize carbon papers. The basic assumptions are overlapping fibers which are long compared to the sample size. Furthermore it is assumed that the GDL is homogeneous on macroscopic view and isotropic in the $\mathrm{x}, \mathrm{y}$ plane.

The stochastic fiber model was developed further by Thiedmann et al. [51, 52, 53]. An important feature of the planar approach is the assumption of isotropy in $\mathrm{z}$ direction and the validation of their model against 3D tomographic data. They developed a method to get the 
model parameters for a 3D geometry model from one $2 \mathrm{D}$ electron microscopy image. This model was used by Wang et al. [54] for numerical transport simulations on the cathode side of a PEFC using CFD.

In our work we simulate the mass transport through a virtual GDL. Its geometry was generated by the 3D stochastic model from Thiedmann et al. [51] with their extended binder model $[52,53]$. The chosen geometry model is favorable for virtual material design because of its planar oriented structure which is nevertheless validated against 3D data.

Therefore, we developed an approach of compression which is planar oriented. We are able to estimate the error caused by the quasi-2D simplification. We simulated the mass transport through the GDL using the LB method and we characterized the GDL by evaluating permeability and tortuosity, both through-plane and in-plane. We applied our methods to uncompressed and compressed virtual geometries. Because of the stochastic nature of the generated microstructure we simulated the mass transport on 25 realizations of the virtual geometry, combined with 4 binder models.

Hao and Cheng [55] calculated also the effect of compression by applying LB simulations on fiber based GDLs and they achieved good agreement with measurements and heuristic trends. Their way of creating the stochastic geometry is different from ours in two aspects.

First, Hao and Cheng created their virtual geometry from the Schladitz model [49] whereas our approach is based on the model from Thiedmann [51] and its extension regarding the binder $[52,53]$.

Second, the more pronounced difference is the way of how the microstructure is compressed. Hao and Cheng reconstructed a new virtual geometry once the porosity was calculated from the rate of compression. They generated the microstructure by applying their reconstruction method with changed porosities and fiber thicknesses according to the compression.

In our work we compress a given microstructure in a geometric way which represents the physical process of compression.

The statistical spread caused by the geometry model has to be separated from the effects due to the compression approach. We introduce a method based on the Kozeny-Carman equation to predict the effect of compression on the permeability. With this approach it was possible to separate the impact of the compression. This allows a validation of the compression model despite of large statistical spread due to the stochastic geometry.

\section{Methods}

Based on the planar oriented geometry model we developed a compression model which is planar oriented, too. This approach is described in detail in section 2.2. The mass transport is simulated with the Lattice Boltzmann (LB) method as presented in section 2.3.

\subsection{Stochastic geometry model}

The virtual geometry of the microstructure of our transport simulations are based on the model of Thiedmann et al. [51]. This model describes fiber based GDL material very well by a simple approach which makes it a promising candidate for virtual material design [56]. The fibers of paper type GDLs are almost oriented in planes which leads to the basic assumption of the Thiedmann model [51]. The fibers of the virtual geometry are created layer-wise by a 2D Poisson line process. Its parameter can be obtained from one SEM image of the GDL. The three dimensional virtual fiber structure was shown to be stochastic equivalent with real 3D material [51]. The remaining solid material to be inserted for achieving the known porosity of $78 \%$ is filled with binder. For this purpose randomly chosen pores built from fibers in a plane are filled with binder material according to Thiedmann's binder model $[52,53]$ until the desired porosity is reached. The binder is dilated in $\mathrm{z}$ direction up to the thickness of the fiber layer.

The method of filling a particular pore with binder either partial or completely is depicted in fig. 1. Given a pore built by some fibers the pore is filled from the border to the center with a constant radius. Fig. 1 shows an example for radii $b_{r}=6 \mu \mathrm{m}, 18 \mu \mathrm{m}, 30 \mu \mathrm{m}$ and $\infty$ which indicates a completely filled pore. Some basic transport simulations on this kind of geometries were presented by Froning et al. [56] where 25 realizations were chosen because of the stochastic nature of the geometry data. The geometry was implemented into a coarse lattice where one fiber layer was represented by 5 lattice layers - all alike which indicates the real fibers being represented as square fibers $(5 \times 5)$ in the virtual geometry.

\subsection{Compression model}

The simplicity of the implemented fiber model induced the demand for a compression model of a similar level of abstraction. Because of the two dimensional approach of the fiber geometry, the compression model was developed also in a two dimensional manner. The basic idea of this approach is that two crossing fibers of adjacent fiber layers penetrate each other when they 
are compressed. This situation is shown in fig. 2. In this diagram the green and red images are representing adjacent fiber layers. Given the chosen implementation already introduced by Froning et al. [56] the two fiber layers are represented by 10 lattice layers. This implementation can be compressed by $10 \%$ by merging the two adjacent lattice layers into one layer. The result is shown in part (c) of fig. 2. In this way 10 lattice layers are compressed to 9 lattice layers. The procedure is repeated stepwise as shown in fig. 3 . The $10 \%$ section of the diagram shows that the principle described above is applied to every second pair of adjacent fiber layers. Applying the similar method to the other pairs of fiber layers leads to a compression of $20 \%$. The merged lattice layers are colored blue in fig. 3. The same method can be repeated by merging new lattice layers as shown in fig. 3 , sections labeled with $30 \%$ to $50 \%$. The $50 \%$ compressed geometry is the extreme case of the compression model. In this case the virtual geometry consists only of merged lattice layers - represented by the black and blue colors of the lines in fig. 3 .

When a GDL with thickness $d_{0}$ is compressed by a compression level $x$ the resulting porosity is

$$
\epsilon_{x}=1-\left(1-\epsilon_{0}\right) \cdot \frac{d_{0}}{d_{x}}
$$

with the porosity $\epsilon_{0}$ of the uncompressed geometry and the thickness $d_{x}$ of the compressed geometry.

The porosity of the virtual geometry compressed by the algorithm described above can be calculated by counting the fluid and solid lattice sites of the generated geometry. Similar, as in our studies in [56], we investigated on 25 realizations of 4 binder models. We compressed those 100 virtual geometries by 10, 20, 30,40 and $50 \%$. The deviations of the porosity of the compressed geometries from eq. (1) are lower than 2.5 / 4.5 / 6.5 / 9 / $12 \%$ for the chosen compression levels - all values averaged over 100 geometries.

\subsection{Transport simulations}

A virtual geometry - both original and the compressed cases - is represented by a series of black/white (BW) images specifying the solid regions as black color and the fluid regions as white [56]. Every image represents a piece of the GDL in its planar orientation with $1.5 \mu \mathrm{m}$ thickness. The situation is depicted in fig. 4 . We are using a lattice of 512 x 512 x 130 units to represent a GDL of $0.77 \mathrm{~mm} \times 0.77 \mathrm{~mm}$ size and $195 \mu \mathrm{m}$ thickness. For the calculation of mass transport a simulation domain is wrapped around the section of the GDL with free space upstream and downstream. This allows the specification of physical meaningful boundary conditions for the gas flow as summarized in table 1. That results in the specification of a constant velocity profile of hydrogen at the inlet boundary (Dirichlet boundary condition). At the outlet a constant pressure was assumed which is equivalent to Neumann boundary conditions on the velocity. At low Reynolds numbers below $10^{-3}$ - related to the fiber diameter - it was found that 10 lattice sites of free space upstream and downstream is sufficient to observe the flow through the GDL undisturbed by the boundary conditions at the inlet and outlet. That extends the lattice to 512 × 512 × 150 units for through-plane simulations in the uncompressed GDL and $532 \times 512 \times 130$ units for in-plane simulations. At the side boundaries of the GDL section no-slip wall boundary conditions were applied. The decision against periodic boundary conditions was made due to the underlying stochastic fiber model. The stochastic model assumes infinite long fibers. This leads to a stochastic parameter which determines the geometric microstructure. Periodic boundary conditions would cut the fibers to the size of the simulation domain. Also a regular structure of the size of the domain would be overlaid which affects the validity of the stochastic model.

\subsection{Kozeny-Carman trend}

To evaluate the capability of the compression model we refer to the Kozeny-Carman equation

$$
\kappa=\frac{\varepsilon}{K_{c}}\left(\frac{V_{p}}{S_{p}}\right)^{2}
$$

The Kozeny-Carman eq. (2) estimates the permeability $\kappa$ of a porous structure from geometric properties and a remaining so-called Kozeny constant $K_{c}$. The porosity $\varepsilon$, the total volume $V_{p}$, and the inner surface of the solid structure $S_{p}$ can be obtained from a given microstructure. The Kozeny constant $K_{c}$ represents the shape of the objects of which the solid structure is built from. The value of this constant cannot be calculated straightforward for complex microstructures. Kaviany [57] reported equations for the Kozeny constant for packed beds of spheres depending of the diameter of the spheres and the porosity of the structure but he admitted a limited applicability of the Kozeny-Carman equation for the calculation of the permeability of a porous structure. Heijs and Lowe [58] calculated the permeability and the Kozeny constant from Lattice Boltzmann calculations on the porous structure of clay soil. They reported a good agreement of their calculated permeabilities with measurements for random packing of spheres. But for real structures they observed dependencies of the calculated permeabilites from the resolution of the images 
representing their geometry and from the resolution of their lattice.

For complex geometries it is known that the Kozeny constant is related to the tortuosity $\tau$ [59]. This leads to

$$
K_{c}=\tau \cdot \tilde{K}_{c}
$$

where $\tilde{K}_{c}$ now depends only on the shape of the geometry. Applied to eq. (2) this leads to the relationship

$$
\kappa \cdot \tau \sim \varepsilon \cdot\left(\frac{V_{p}}{S_{p}}\right)^{2}
$$

which allows us to define a Kozeny-Carman trend

$$
\left.\kappa \cdot \tau\right|_{x \%}=\left.\left.\kappa \cdot \tau\right|_{0 \%} \cdot \varepsilon\left(\frac{V_{p}}{S_{p}}\right)^{2}\right|_{x \%}\left|\varepsilon\left(\frac{V_{p}}{S_{p}}\right)^{2}\right|_{0 \%}
$$

In eq. (5) the symbols $\kappa, \tau, \varepsilon, V_{p}$, and $S_{p}$ specify properties of the uncompressed or compressed geometry depending on whether $\left.\right|_{0 \%}$ or $\left.\right|_{x \%}$ is written rightmost to the symbol. The Kozeny-Carman trend estimates $\kappa \cdot \tau$ of a compressed geometry by evaluating geometric properties of the microstructure and relating it to the calculated permeability and tortuosity of the uncompressed geometry. We used eq. (5) to validate the simulations on compressed geometries.

\subsection{Accuracy of the results}

The fibers of the stochastic model are represented in a coarse grid with 5 lattice sites within a fiber diameter. A quadratic profile of the fibers is used according to Thiedmann et al. [51]. This resolution is according to the resolution of the synchrotron data which was used to validate the stochastic model [51].

From studies on the accuracy of the calculated permeability by Brinkmann et al. [60] it can be concluded that the error in the permeability of our simulations in the GDL structure is below $10 \%$.

The error inherent to the compression model because of the simplified approach shown in fig. 2 is below $6.5 \%$ as argued in section 2.2. With the deviation of $\kappa \cdot \tau$ from the Kozeny-Carman trend it can be estimated that the error in $\kappa \cdot \tau$ caused by the compression model is in the same order of magnitude as the model-related error in the geometry. With this approach we are able to separate the influence of the compression from the influence of other parameters of the complex simulation, e.g. LB schemes, discretization, boundary conditions, or even size effects.

The accuracy of the LB simulation itself can be increased when the underlying geometry is represented more accurate by a finer lattice. This was shown by
Thomas et al. [61] who investigated the effect of fiber resolution on on LB simulations.

In the present article the transport simulations are based on a simplified geometry model with a coarse resolution [51]. The special approach of compressing this type of geometry is dedicated to the assumptions of the stochastic geometry. As a conclusion it is not useful to enhance the spatial resolution in our case.

The accuracy of the results is in the same order of magnitude as the coarse geometry and the simple approach of compression. Furthermore, the KozenyCarman trend (eq. (5)) allows to judge the simulations on compressed geometries in relation to the uncompressed geometry - assuming the error in the calculated permeability being dominated by systematic effects.

\section{Results}

\subsection{Transport simulations}

For our LB simulations we used the open source software Palabos [62]. We chose the BGK method and the D3Q19 discretization scheme [56, 16, 63]. As a result we get fields of

$$
f_{i}(\xi, \vartheta), i=0, \ldots, 18
$$

where $f_{i}(\xi, \vartheta)$ specifies the probability of finding a gas molecule at the position $\xi$ and time $\vartheta$ transported in the $i$-th direction of the D3Q19 scheme to the neighbored voxel within a fixed time step $\delta t$. Velocity vectors can be calculated from a combination of the $f_{i}$ which results in a field of velocities throughout the simulation domain $[16,63]$. In fig. 5 a section of the GDL is presented after convergence of the transport simulation to steady state. The diagram shows the simulated section of an uncompressed GDL - a realization of binder type B (see fig. 1) with binder radius $b_{r}=18 \mu \mathrm{m}$. In the fig. 5 the gas flows from the bottom to the top. A plane perpendicular to the GDL was chosen for graphical post processing. Path lines of fixed length starting from this post processing plane were calculated in upstream and downstream direction. They were colored with the velocity of the fluid. In this way it is demonstrated that the flow is very irregular in its local directions and velocities.

\subsection{Permeability and Tortuosity}

For studies on virtual geometries we calculated two characteristic numbers for evaluation. Like in [56] we calculated the permeability from Darcy's law

$$
\kappa=-\frac{q \cdot \mu}{\nabla P}
$$


where $q$ is the flux through the porous structure, $\mu$ is the dynamic viscosity of the fluid. $\nabla P$ represents the pressure drop along the entire porous structure in flow direction, i.e. between the planes where the fluid enters the porous structure and where it leaves. We calculated the tortuosity in a simplified way as presented by Koponen et al. [64]

$$
\tau=\frac{\langle|v|\rangle}{\left\langle v_{x}\right\rangle}
$$

In eq. (8) \langle\rangle denotes the spatial average over the region of interest, that section namely, where the simulated GDL is situated. This allows the calculation of the tortuosity from the velocity field where $|v|$ is the absolute value of the velocity and $v_{x}$ is its component in flow direction. The tortuosity calculated by eq. (8) was proven to be correct for capillary systems [64]. For general porous structure this approach is an approximation.

\subsection{Domain size dependency}

To overcome the size effect of the simulation domain, through-plane simulations were run at virtual geometries of smaller sections - $100 \times 100 \times 150$, $200 \times 200 \times 150,300 \times 300 \times 150$, and $400 \times 400 \times 150$ instead of $512 \times 512 \times 150$ lattice units. The statistical spread of the tortuosity computed from simulations in 25 independent realizations of the stochastic geometry of each size was compared. It was found that a section of 300 x 300 is sufficient large in all cases. Depending on the binder model even smaller sections of $100 \times 100$ were large enough. The size of the chosen section of the GDL - images of 512 x 512 pixels - represents a section of $0.77 \mathrm{~mm} \times 0.77 \mathrm{~mm}$.

Measurements of permeability on GDL material of Toray were published by Gostick et al. [65, 66]. Hussaini and Wang reviewed the available measured data including Gostick - and completed the data with own measurements [6]. The measured through-plane permeability of Toray 090 is ranging from 4.4 to $12.4 \mu \mathrm{m}^{2}$. The values were obtained from different GDL thicknesses, different porosity and different levels of compression. The permeabilites calculated from our simulations in virtual geometries are in the right order of magnitude as shown in table 2. Simulations on uncompressed geometries and $10 \%$ compression were compared with experimental values from literature.

The tortuosity of random overlapping fiber structures was calculated analytically by Tomadakis and Robertson [59]. Their approach of 2-dimensiomal overlapping fiber structures is equivalent to the Thiedmann model of the fiber based geometry without binder. For such structures Tomadakis and Robertson calculated the tortuosity by eq. (9).

$$
\tau=\left(\frac{1-\varepsilon_{p}}{\varepsilon-\varepsilon_{p}}\right)^{\alpha}
$$

In this equation $\varepsilon$ is the porosity of the fiber structure, $\varepsilon_{p}=0.11, \alpha=0.785$ for through-plane flow and $\alpha=0.521$ for in-plane flow. Table 3 shows a very good agreement between the values according eq. (9) and the tortuosities simulated in a fiber structure without binder. The deviation between the simulations and eq. (9) of about $4 \%$ is due to the discretization of the fiber structure.

\subsection{Results on virtual geometries}

Transport simulations were run on 4 binder models as depicted in fig. 1, A: $b_{r}=6 \mu \mathrm{m}, \mathrm{B}: b_{r}=18 \mu \mathrm{m}, \mathrm{C}: b_{r}=$ $30 \mu \mathrm{m}$, and D: $b_{r}=\infty$ which is synonym to filled pores. Because of the anisotropy of the GDL transport simulations were performed through-plane and in-plane. 25 realizations of the geometry were generated from each of the 4 binder models, resulting in 100 virtual geometries in total. It has to be admitted that the flow simulation through the compressed geometries did not converge in some cases of the unrealistic type $\mathrm{D}$ geometries especially when large pores filled with binder blocked the fluid flow massively. In such cases less than 25 realizations were considered. Table 4 shows the summarized results of the permeability and tortuosity calculated from through-plane simulations on the uncompressed and compressed virtual geometries.

The table contains the average values and variation coefficients of the tortuosities and permeabilities. The variation coefficient $V=\sigma / \bar{x}$ with the standard deviation $\sigma$ and the mean value $\bar{x}$ is a dimensionless measure of the statistical spread which can be used to compare samples with different mean values [67]. The type D simulations - GDLs with completely filled pores show larger variation coefficients of the tortuosity than the others. This is conform to the intuitive expectation because the filled pores may block the gas flow locally.

Table 5 shows the values for in-plane simulations. The difference in the variation coefficient due to different binder types was not observed on the in-plane simulations. This effect is induced by the layer-wise construction of the geometric model. The binder is always oriented in-plane.

Fig. 6 shows the tortuosities of the through-plane simulations on the uncompressed geometries. The statistical spread is large compared to the systematic 
change of the absolute values according to the binder model. The type D model shows clearly a larger statistical spread than the others. Also the statistical spread of the permeability is large as shown in fig. 7 for throughplane simulations. While the tortuosity is increasing with the binder radius the permeability is decreasing.

Fig. 8 shows the calculated permeabilities of binder model $\mathrm{B}$, through-plane simulation, where the geometry is compressed by $10,20,30,40$, and $50 \%$. The $30 \%$ curve represents a compression level which was observed in fuel cells operated at Forschungszentrum Jülich. It is clearly shown in fig. 8 that the compression of the geometry has a systematic influence on the through-plane permeability in every realization of the virtual geometries. It can also be observed that the variance across the stochastic geometries is significant at every compression level. The variance represents the inhomogeneous nature of the stochastic geometry. We investigated also in transport simulations in smaller domains [56]. We observed that the simulation domain of $512 \times 512$ is large enough to prevent the mean values of the results from size effects.

In order to check if the number of realizations causes the scattering effects of the calculated values regarding the number of realizations we summarized the mean values and the variation coefficients of the most sensitive value - which is the permeability - for different numbers of realizations in table 6. As the worst case of the geometry we chose through-plane simulations on geometries with type D binder. The high sensitivity of the permeability compared to the other binder types and to the tortuosity is depicted by the high values of the variation coefficient in tables 4 and 5 . It was shown that about 20 realizations are sufficient to keep the variation coefficient almost constant.

\section{Discussion}

The compression model cannot be validated by merely evaluating the absolute values of the permeability because of the large statistical spread. We refer also to the Kozeny-Carman equation as shown in section 2.4. The results of through-plane and in-plane simulations are discussed in section 4.1 and section 4.2.

\subsection{Through-plane simulations}

The gas flow was simulated on uncompressed geometries and also on compressed geometries with compression levels of $10,20,30,40$, and $50 \% .100$ realizations of virtual geometries -25 realizations of every binder model A, B, C, and D - were considered which are 600 virtual geometries in total. Transport simulations were performed through-plane and in-plane.

Fig. 9 shows the averaged results of $\kappa \cdot \tau$ for throughplane simulations. The solid lines represent the KozenyCarman trend estimated from eq. (5). The symbols represent transport simulations on the compressed geometries. It can be observed that the simulated values of $\kappa \cdot \tau$ agree very well with the Kozeny-Carman trend.

The deviations of the through-plane simulations presented in fig. 9 are also shown as relative deviations in table 7 . The numbers in this table are average values taken from 25 realizations each. The realistic conditions are compression levels up to $30 \%$ as observed at Forschungszentrum Jülich. The virtual geometry is expected to be realistic for the binder models A, B, or C, i.e. pores are not completely filled with binder. For these conditions the averaged deviation of $\kappa \cdot \tau$ from the Kozeny-Carman trend is below $11.4 \%$ (through-plane).

The deviation of the simulated $\kappa \cdot \tau$ from the values estimated from eq. (5) are slightly larger in two cases. One is the extreme case of $50 \%$ compression. The deviation is clearly higher than for smaller compression levels. The $50 \%$ compression is structural different from the others because in this case some fibers come into contact which were not adjacent in the uncompressed geometry. This structural change leads to large deviations of the simulations from the Kozeny-Carman trend. The reason is not only a local change of the topology but also a change of the inner surface $S_{p}$ in eq. (5).

The other extreme case is the simulation on the binder model $\mathrm{D}$ representing the binder model with filled pores. This is also an extreme case - it is expected to be far away from real GDL structures of paper type $[52,56]$. In table 7 the deviation of the $50 \%$ compressed binder type D simulations from the KozenyCarman trend is significantly lower than for the other cases. The reason is a smaller number of realizations - as shown in table 4 - which were considered for the evaluation because some realizations did not converge as mentioned in section 3.4.

Though the simulated section of the GDL - represented by 512 x 512 lattice sites - is large enough to overcome size effects in the planar oriented direction (section 3.3) the through-plane extent might limit the accuracy of the results. In section 2.3 we justified the presence of free space upstream and downstream of the GDL structure to specify physical meaningful boundary conditions. The re-arrangement of the mass flow at the boundaries of the GDL takes place in a region of about 5 voxels in the free space but also in a region of about 5 voxels inside the GDL at the chosen operating conditions (Reynolds number below $10^{-3}$ ). In 
uncompressed GDLs we found that the thickness of the GDL is sufficient large to calculate permeabilites and tortuosities which do not change when the thickness of the GDL is increased. But under compression the thickness of the GDL decreases, so does the lattice. Consequently, the region of uncertainty inside the GDL - up to 5 voxels on both sides - becomes more pronounced compared to the uncompressed structure.

\subsection{In-plane simulations}

The results of $\kappa \cdot \tau$ from in-plane simulations are shown in fig. 10. Table 8 contains the relative deviations of the in-plane simulation from the KozenyCarman trend.

Compared to the through-plane simulations in fig. 9 the absolute deviations from the Kozeny-Carman trend defined by eq. (5) don't show a preference regarding the binder model. The reason is that the filled pores can block significantly through-plane flow but in-plane the fluid flows along the binder.

The thickness of the GDL may possibly limit the accuracy of the in-plane simulations. The size of the domain (512 by 512 ) is large enough for through-plane simulations which was shown by simulating gas flow through GDL sections of different size (section 3.3). For in-plane simulations the side walls are much closer: 195 lattice units in the uncompressed simulations, and 117, $105,92,80$, and 67 units for compression levels from 10 to $50 \%$. Flow simulations in such small regions do not represent material properties which are independent from the conditions at the boundaries of the simulation domain.

This is conform to the simulations results from Becker et al. [68]. They measured the permeability of Toray GDLs through-plane and in-plane. Transport simulations - through-plane and in-plane - were applied to the microstructure obtained from phase contrast tomographic microscopy. Becker at al. achieved a good agreement of their simulations with their through-plane measurements but they observed deviations from the measurements in case of in-plane simulations. Becker admitted that the in-plane measurements were performed on several GDL layers stacked on each other to get reliable measurements. This experimental procedure was already reported by Hussaini et al. [6]. This experimental size effect correlates to the size effect mentioned in section 3.3 .

It can be concluded that the in-plane mass transport cannot be described properly by material properties. In narrow regions of compressed GDLs the conditions at the wall boundaries may affect the mass transport significantly.

\section{Conclusion}

In our paper we presented a stochastic model of fiber based GDL microstructures. Its simplicity favors it for virtual material design. For the existing planar oriented geometry model we developed a compression model which is of a similar level of abstraction as the underlying fiber model. We simulated single component fluid transport through 100 realizations of the virtual geometries both uncompressed and compressed. This simulations enable us to evaluate the statistical impact on effective material properties for gas flow in paper-type GDLs used in HT-PEFCs.

Four types of binder models were considered. The compression leads to systematic changes in the calculated permeability and tortuosity. The statistical spread is in the same order of magnitude as the systematic change caused by the compression.

Compression of the microstructure leads to lower permeabilities. The effect resulting from the transport simulations on the compressed geometries was compared with the values estimated from the uncompressed geometries using the Karman-Cozeny equation. The product of permeability and tortuosity $\kappa \cdot \tau$ is a characteristic which describes very well the behavior of effective transport properties according to the Karman-Cozeny equation. For configurations of practical interest the agreement of the through-plane simulations was very well which approves the usability of the compression model for the desired material. The accuracy of the simulations on a $512 \times 512 \times 150$ lattice is in the same range as the geometric accuracy of the underlying stochastic model.

The thickness of the GDL was too small to prevent the simulations from a size effect introduced by the wall boundaries. This has to be considered when results shall be applied to larger scales. To obtain in-plane material properties independent from the GDL thickness the transport simulations need to be run in larger regions.

\section{Acknowledgements}

This research is funded by the German Federal Ministry of Education and Research grant 03MS507. Simulations were performed on hardware of the Jülich $\mathrm{Su}-$ percomputing Centre. 


\section{References}

[1] M. F. Mathias, J. Roth, J. Fleming, W. Lehnert, Diffusion media materials and characterisation, in: W. Vielstich (Ed.), Handbook of Fuel Cells, Vol. 3, Wiley, 2003, pp. 517-537.

[2] R. P. O'Hayre, S.-W. Cha, W. G. Colella, F. B. Prinz, Fuel Cell Fundamentals, 2nd Edition, Wiley, New York, 2009.

[3] C. Hartnig, L. Jörissen, J. Kerres, W. Lehnert, J. Scholta, Polymer electrolyte fuel cells, in: M. Gasik (Ed.), Materials for fuel cells, 1st Edition, Woodhead publishing, Cambridge, 2008, pp. $101-184$

[4] Y. Wang, K. S. Chen, Modeling of polymer electrolyte membrane fuel-cell components, in: D. Stolten, B. Emonts (Eds.) Fuel Cell Science and Engineering, 1st Edition, WILEY-VCH, Weinheim, 2012, pp. 839-878.

[5] W.-K. Lee, C.-H. Ho, J. W. van Zee, M. Murthy, The effects of compression and gas diffusion layers on the performance of a pem fuel cell, J. Power Sources 84 (1999) 45-51.

[6] I. S. Hussaini, C. Y. Wang, Measurement of relative permeability of fuel cell diffusion media, J. Power Sources 195 (2010) 3830-3840.

[7] V. Radhakrishnan, P. Haridoss, Differences in structure and property of carbon paper and carbon cloth diffusion media and their impact on proton exchange membrane fuel cell flow field design, Mater. Des. 32 (2011) 861-868.

[8] K. K. Poornesh, Y.-J. Sohn, G.-G. Park, T.-H. Yan, Gasdiffusion layer's structural anisotropy induced localized instability of nafion membrane in polymer electrolyte fuel cell, Int. J. Hydrogen Energy 37 (2012) 15339-15349.

[9] N. Parikh, J. S. Allen, R. S. Yassar, Microstructure of gas diffusion layers for PEM fuel cells, Fuel Cells 12 (2012) 382-390.

[10] I. Manke, C. Hartnig, M. Grünerbel, W. Lehnert, N. Kardjilov, A. Haibel, A. Hilger, J. Banhart, H. Riesemeier, Investigation of water evolution and transport in fuel cells with high resolution synchrotron x-ray radiography, Appl. Phys. Lett. 90 (2007) 174105 .

[11] T. Sasabe, P. Deevanhxay, S. Tsushima, S. Hirai, Investigation on the effect of microstructure of proton exchange membrane fuel cell porous layers on liquid water behavior by soft x-ray radiography, J. Power Sources 196 (2011) 8197-8206.

[12] M. Kvesić, U. Reimer, D. Froning, L. Lüke, W. Lehnert, D. Stolten, $3 \mathrm{~d}$ modeling of a $200 \mathrm{~cm}^{2}$ ht-pefc short stack, Int J. Hydrogen Energy 37 (2012) 2430-2439.

[13] M. Kvesić, U. Reimer, D. Froning, L. Lüke, W. Lehnert, D. Stolten, 3d modeling of an ht-pefc stack using reformate gas, Int. J. Hydrogen Energy 37 (2012) 12438-12450.

[14] A. C. Olesen, T. Berning, S. K. Kær, The effect of inhomogeneous compression on water transport in the cathode of a proton exchange membrane fuel cell, J. Fuel Cell Sci. Technol. 9 (2012) 031010-1 - 031010-7.

[15] P. Chippar, H. Ju, Three-dimensional non-isothermal modeling of a phosphoric acid-doped polybenzimidazole (pbi) membrane fuel cell, Solid State Ionics 225 (2012) 30-39.

[16] S. Succi, The Lattice Boltzmann Equation, Oxford University Press, Oxford, 2001.

[17] M. A. Khan, B. Sunden, J. Yuan, Analysis of multi-phase transport phenomena with catalyst reactions in polymer electrolyte membrane fuel cells - a review, J. Power Sources 196 (2011) 7899-7916.

[18] X. Shan, H. Chen, Lattice boltzmann model for simulating flows with multiple phases and components, Phys. Rev. E 47 (3) (1993) 1815-1819.

[19] A. J. Joshi, K. N. Grew, A. A. Peracchio, W. K. S. Chiu, Lattice boltzmann modeling of $2 \mathrm{~d}$ gas transport in a solid oxide fuel cell anode, J. Power Sources 164 (2007) 631-638.
[20] S. Chen, Z. Liu, Z. Tian, B. Shi, C. Zheng, A simple lattice boltzmann scheme for combustion simulation, Computers and Mathematics with Applications 55 (2008) 1424-1432.

[21] P. Rama, Y. Liu, H. Ostadi, K. Jiang, Y. Gao, X. Zhang, R. Fisher, M. Jeschke, Multiscale modeling of single-phase multicomponent transport in the cathode gas diffusion layer of a polymer electrolyte fuel cell, Energy Fuels 24 (2010) 31303143.

[22] C. K. Aidun, J. R. Clausen, Lattice-boltzmann method for complex flows, Annu. Rev. Fluid Mech. 42 (2010) 439-472.

[23] L. Hao, P. Cheng, Pore-scale simulations on relative permeabilities of porous media by lattice boltzmann method, Int. J. Heat Mass Transfer 53 (2010) 1908-1913.

[24] P. P. Mukherjee, Q. Kang, R. Mukundan, R. L. Borup, Numerical modeling of two-phase behavior in the pefc gas diffusion layer, ECS Trans. 26 (1) (2010) 97-106.

[25] J. Tölke, S. Freudiger, M. Krafczyk, An adaptive scheme using hierarchical grids for lattice boltzmann multi-phase flow simulations, Comput. Fluids 35 (2006) 820-830.

[26] D. d'Humieres, I. Ginzburg, Viscosity independent numerical errors for lattice boltzmann models: From recurrence equations to "magic" collision numbers, Computers and Mathematics with Applications 58 (2009) 823-840.

[27] I. Ginzburg, D. d'Humieres, Multireflection boundary conditions for lattice boltzmann models, Phys. Rev. E 68 (6) (2003) 66614-1-30.

[28] M. Hecht, J. Harting, Implementation of on-site velocity boundary conditions for $\mathrm{d} 3 \mathrm{q} 19$ lattice boltzmann simulations, J. Stat. Mech, (2010) 1018+23.

[29] M. Hossain, S. Z. Islam, P. Pollard, Investigation of species transport in a gas diffusion layer of a polymer electrolyte membrane fuel cell through two-phase modelling, Renewable Energy 51 (2013) 404-418.

[30] P. K. Sinha, C.-Y. Wang, Pore-network modeling of liquid water transport in gas diffusion layer of a polymer electrolyte fuel cell, Electrochim. Acta 52 (2007) 7936-7945.

[31] J. LaManna, S. G. Kandlikar, A critical review on water transport models in gas diffusion media of PEM fuel cell, in: Proc. 6th Int. ASME Conf. on Nanochannels, Microchannels and Minichannels, no. ICNMM2008-62201, ASME, Darmstadt, Germany, 2008.

[32] G. Inoue, Y. Matsukama, M. Minemoto, Evaluation of twophase condition and mass transfer in gdl with pore network model, in: Proc. FuelCell2009, 2009.

[33] G. Luo, Y. Ji, C.-Y. Wang, P. K. Sinha, Modeling liquid water transport in gas diffusion layers by topologically equivalent pore network, Electrochim. Acta 55 (19) (2010) 5332-5341. doi:10.1016/j.electacta.2010.04.078.

[34] Y. Ma, J. Li, Z. Zhang, X. Wang, Evaluating breakthrough pressure in gas diffusion layers of proton exchange membrane fuel cells, J. Therm. Sci. 19 (2010) 459-464.

[35] J. T. Gostick, Random pore network modeling of gdls using voronoi and delaunay tesselations, ECS Trans. 41 (1) (2011) $125-130$.

[36] C. Tötzke, I. Manke, C. Hartnig, R. Kuhn, H. Riesemeier, J. Banhart, Investigation of carbon fiber gas diffusion layers by means of synchrotron x-ray tomography, ECS Trans. 41 (1) (2011) 379-386.

[37] F. Büchi, J. Eller, F. Marone, M. Stampanoni, Determination of local gdl saturation of the pore level by in situ synchrotron based X-ray tomographic microscopy, ECS Trans. 33 (1) (2010) 1397-1405.

[38] R. Flückiger, F. Marone, M. Stampanoni, A. Wokaun, F. N. Büchi, Investigation of liquid water in gas diffusion layers of polymer electrolyte fuel cells using x-ray tomographic mi- 
croscopy, Electrochim. Acta 56 (2011) 2254-2262.

[39] T. Rosén, J. Eller, J. Kang, N. I. Prasianakis, J. Mantzaras, F. N. Büchi, Saturation dependent effective transport properties of pefc gas diffusion layers, J. Electrochem. Soc. 159 (9) (2012) F536-F544.

[40] X.-D. Niu, T. Munekata, S.-H. Hyodo, K. Suga, An investigation of water-gas transport processes in the gas-diffusion layer of a pem fuel cell by a multiphase multi-relaxation-time lattice boltzmann model, J. Power Sources 172 (2007) 542-552.

[41] H. Ostadi, P. Rama, Y. Liu, R. Chen, X. Zhang, K. Jiang, Nanotomography based study of gas diffusion layers, Microelectron. Eng. 87 (2010) 1640-1642.

[42] H. Ostadi, P. Rama, Y. Liu, R. Chen, X. X. Zhang, K. Jiang, 3d reconstruction of a gas diffusion layer and a microporous layer, J. Membr. Sci. 351 (2010) 69-74.

[43] H. Ostadi, P. Rama, R. Chen, X. Zhang, Y. Gao, K. Jiang, 3d visualization and characterization of nano structured materials, in: 2011 11th IEEE International Conference on Nanotechnology, 2011, pp. 770-775.

[44] P. Rama, Y. Liu, R. Chen, H. Ostadi, K. Jiang, Y. Gao, X. Zhang, D. Brivio, P. Grassini, A numerical study of structural change and anisotropic permeability in compressed carbon cloth polymer electrolyte fuel cell gas diffusion layers, Fuel Cells 11 (2) (2011) 274-285.

[45] Y. Gao, X. X. Zhang, P. Rama, Y. Liu, R. Chen, H. Ostadi, $\mathrm{K}$. Jiang, Modeling fluid flow in the gas diffusion layers in pemfc using the multiple relaxation-time lattice boltzmann method, Fuel Cells 12 (2012) 365-381.

[46] Y. Gao, X. Zhang, P. Rama, R. Chen, H. Ostadi, K. Jiang, An improved mrt lattice boltzmann model for calculating anisotropic permeability of compressed and uncompressed carbon cloth gas diffusion layers based on x-ray computed micro-tomography, J. Fuel Cell Sci. Technol. 9 (2012) 041010-1-10.

[47] G. Gaiselmann, R. Thiedmann, I. Manke, W. Lehnert, V. Schmidt, Stochastic 3d modeling of fiber-based materials, Comput. Mater. Sci. 59 (2012) 75-86.

[48] M. M. Tomadakis, S. V. Sotirchos, Ordinary and transition regime diffusion in random fiber structures, in: AIChE J. [59], pp. 397-412 (1993) 397-412.

[49] K. Schladitz, S. Peters, D. Reinel-Blitzer, A. Wiegmann, J. Ohser, Design of acoustic trim based on geometric modeling and flow simulation for non-woven, Comput. Mater. Sci. 38 (2006) 56-66

[50] V. P. Schulz, J. Becker, A. Wiegmann, P. P. Mukherjee, C.Y. Wang, Modeling of two-phase behavior in the gas diffusion medium of PEFCs via full morphology approach, J. Electrochem. Soc. 154 (2007) B419-B426.

[51] R. Thiedmann, F. Fleischer, C. Hartnig, W. Lehnert, V. Schmidt, Stochastic 3d modeling of the gdl structure in pemfes based on thin section detection, J. Electrochem. Soc. 155 (4) (2008) B391-B399.

[52] R. Thiedmann, C. Hartnig, I. Manke, V. Schmidt, W. Lehnert, Local structural characteristics of pore space in gdls of pem fuel cells based on geometric 3d graphs, J. Electrochem. Soc. 156 (11) (2009) B1339-B1347.

[53] R. Thiedmann, G. Gaiselmann, W. Lehnert, V. Schmidt, Stochastic modeling of fuel-cell components, in: D. Stolten, B. Emonts (Eds.), Fuel Cell Science and Engineering, 1st Edition, WILEY-VCH, Weinheim, 2012, pp. 669-702.

[54] Y. Wang, S. Cho, R. Thiedmann, V. Schmidt, W. Lehnert, $\mathrm{X}$. Feng, Stochastic modeling and direct simulation of the diffusion media for polymer electrolyte fuel cells, Int. J. Heat Mass Transfer 53 (2010) 1128-1138.

[55] L. Hao, P. Cheng, Lattice boltzmann simulations of anisotropic permeabilities in carbon paper gas diffusion layers, J. Power
Sources 186 (2009) 104-114.

[56] D. Froning, G. Gaiselmann, U. Reimer, J. Brinkmann, W. Lehnert, V. Schmidt, Stochastic aspects of mass transport in gas diffusion layers, J. Electrochem. Soc. submitted.

[57] M. Kaviany, Principles of Heat Transfer in Porous Media, 2nd Edition, Springer, 1995.

[58] A. Heijs, C. Lowe, Numerical evaluation of the permeability and the kozeny constant for two types of porous media, Phys. Rev. E 51 (5) (1995) 4346-4353.

[59] M. M. Tomadakis, T. J. Robertson, Viscous permeability of random fiber structures: Comparison of electrical and diffusional estimates with experimental and analytical results, J. Compos. Mater. 39 (2) (2005) 163-188.

[60] J. P. Brinkmann, D. Froning, U. Reimer, V. Schmidt, W. Lehnert, D. Stolten, 3d modeling of one and two component gas flow in fibrous microstructures in fuel cells by using the lattice boltzmann method, ECS Trans. submitted.

[61] M. L. R. Thomas, D. B. Ingham, M. Pourkashanian, Prediction of the permeability of fibrous media using the lattice boltzmann method in conjunction with coarse numerical lattices, Open Transp. Phenom. J. 2 (2010) 80-89.

[62] http://www.palabos.org/ (Nov 14, 2012).

[63] D. Hänel, Molekulare Gasdynamik, Springer, 2004.

[64] A. Koponen, M. Kataja, J. Timonen, Tortuous flow in porous media, Phys. Rev. E 54 (1) (1996) 406-410.

[65] J. T. Gostick, M. W. Fowler, M. D. Pritzker, M. A. Ioannidis, L. M. Behra, In-plane and through-plane gas permeability of carbon fiber electrode backing layers, J. Power Sources 162 (2006) 228-238.

[66] J. T. Gostick, M. W. Fowler, M. A. Ioannidis, M. D. Pritzker, Y. M. Volfkovich, A. Sakars, Capillary pressure and hydrophilic porosity in gas diffusion layers for polymer electrolyte fuel cells, J. Power Sources 156 (2006) 375-387.

[67] L. Sachs, Angewandte Statistik, Springer, 1999.

[68] J. Becker, R. Flückiger, M. Reum, F. N. Büchi, F. Marone, M. Stampanoni, Determination of material properties of gas diffusion layers: Experiments and simulations using phase contrast tomographic microscopy, J. Electrochem. Soc. 156 (2009) B1175-B1181.

\section{Tables and Figures}

Table 1: Operating conditions

\begin{tabular}{ll}
\hline average current density & $1 \mathrm{~A} / \mathrm{cm}^{2}$ \\
\hline volumetric flow rate $\mathrm{H}_{2}$ & $7 \mathrm{ml} / \mathrm{min}$ \\
\hline operating temperature & $160{ }^{\circ} \mathrm{C}$ \\
\hline superficial velocity $\mathrm{H}_{2}$ & $1.8 \cdot 10^{-3} \mathrm{~m} / \mathrm{s}$
\end{tabular}


Table 2: Measured permeabilities on Toray 090 reported by Hussaini et al.[6] vs. simulated values. TP: through-plane configuration, IP: in-plane configuration

\begin{tabular}{|c|c|c|c|}
\hline conf. & comp. & $\begin{array}{c}\text { permeability } \\
/ \mu \mathrm{m}^{2}\end{array}$ & origin \\
\hline \multirow[t]{11}{*}{$\mathrm{TP}$} & $10 \%$ & 9 & cited in [6] \\
\hline & $5 \%$ & 4.4 & cited in [6] \\
\hline & $9 \%$ & $12.4 \pm 0.88$ & measured by [6] \\
\hline & $0 \%$ & $11.7 \pm 0.89$ & sim., binder A \\
\hline & $0 \%$ & $11.2 \pm 1.16$ & sim., binder B \\
\hline & $0 \%$ & $10.5 \pm 1.07$ & sim., binder $\mathrm{C}$ \\
\hline & $0 \%$ & $8.2 \pm 1.44$ & sim., binder D \\
\hline & $10 \%$ & $9.64 \pm 0.76$ & sim., binder $\mathrm{A}$ \\
\hline & $10 \%$ & $9.14 \pm 0.99$ & sim., binder B \\
\hline & $10 \%$ & $8.55 \pm 0.91$ & sim., binder $\mathrm{C}$ \\
\hline & $10 \%$ & $6.59 \pm 1.22$ & sim., binder D \\
\hline \multirow[t]{10}{*}{ IP } & $0 \%$ & 20 & cited in [6] \\
\hline & $9 \%$ & $14.6 \pm 2$ & measured by [6] \\
\hline & $0 \%$ & $17.6 \pm 1.39$ & sim., binder $\mathrm{A}$ \\
\hline & $0 \%$ & $18.0 \pm 1.99$ & sim., binder B \\
\hline & $0 \%$ & $17.8 \pm 1.63$ & sim., binder $\mathrm{C}$ \\
\hline & $0 \%$ & $16.9 \pm 1.68$ & sim., binder D \\
\hline & $10 \%$ & $14.4 \pm 1.17$ & sim., binder A \\
\hline & $10 \%$ & $14.7 \pm 1.66$ & sim., binder B \\
\hline & $10 \%$ & $14.6 \pm 1.37$ & sim., binder $\mathrm{C}$ \\
\hline & $10 \%$ & $13.8 \pm 1.42$ & sim., binder D \\
\hline
\end{tabular}

Table 3: Tortuosity $\tau$ of GDLs without binder

\begin{tabular}{cccc}
\hline No. & porosity & $\tau$ by eq. $(9)$ & $\tau$ simulated \\
\hline 1 & 0.863 & 1.140 & 1.187 \\
2 & 0.867 & 1.135 & 1.184
\end{tabular}

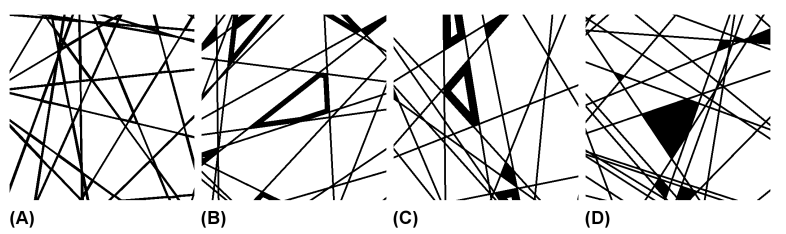

Figure 1: Four types of binder with different binder radii, A) $b_{r}=6$ $\mu \mathrm{m}$, B) $b_{r}=18 \mu \mathrm{m}$, C) $b_{r}=30 \mu \mathrm{m}$, and D) $b_{r}=\infty$
Table 4: Through-plane characteristics of uncompressed and compressed GDLs. BT are binder types A: $b_{r}=6 \mu \mathrm{m}, \mathrm{B}: b_{r}=18 \mu \mathrm{m}$, $\mathrm{C}: b_{r}=30 \mu \mathrm{m}, \mathrm{D}$ : filled pores. $\mathrm{CL}$ is the compression level from 0 to $50 \%$.

\begin{tabular}{|c|c|c|c|c|c|c|}
\hline \multirow[t]{2}{*}{ BT } & \multirow{2}{*}{$\begin{array}{l}\text { CL } \\
1 \%\end{array}$} & \multicolumn{2}{|c|}{ Tortuosity $\tau$} & \multicolumn{2}{|c|}{ Permeability $/ \mu \mathrm{m}^{2}$} & \multirow{2}{*}{$\begin{array}{l}\text { No. } \\
\text { sim. }\end{array}$} \\
\hline & & mean & $\begin{array}{l}\text { var. } \\
\text { coef. }\end{array}$ & mean & $\begin{array}{l}\text { var. } \\
\text { coef. }\end{array}$ & \\
\hline \multirow[t]{6}{*}{$\mathrm{A}$} & 0 & 1.24 & $5.9 \cdot 10^{-3}$ & 11.73 & $7.6 \cdot 10^{-2}$ & 25 \\
\hline & 10 & 1.25 & $6.1 \cdot 10^{-3}$ & 9.64 & $7.9 \cdot 10^{-2}$ & 25 \\
\hline & 20 & 1.27 & $6.3 \cdot 10^{-3}$ & 7.75 & $8.2 \cdot 10^{-2}$ & 25 \\
\hline & 30 & 1.29 & $6.6 \cdot 10^{-3}$ & 5.93 & $8.7 \cdot 10^{-2}$ & 25 \\
\hline & 40 & 1.32 & $6.5 \cdot 10^{-3}$ & 4.33 & $9.5 \cdot 10^{-2}$ & 22 \\
\hline & 50 & 1.37 & $7.5 \cdot 10^{-3}$ & 2.81 & $1.1 \cdot 10^{-1}$ & 20 \\
\hline \multirow[t]{6}{*}{ B } & 0 & 1.27 & $9.4 \cdot 10^{-3}$ & 11.18 & $1.0 \cdot 10^{-1}$ & 25 \\
\hline & 10 & 1.29 & $9.9 \cdot 10^{-3}$ & 9.14 & $1.1 \cdot 10^{-1}$ & 25 \\
\hline & 20 & 1.30 & $1.0 \cdot 10^{-2}$ & 7.32 & $1.1 \cdot 10^{-1}$ & 25 \\
\hline & 30 & 1.33 & $1.1 \cdot 10^{-2}$ & 5.56 & $1.2 \cdot 10^{-1}$ & 25 \\
\hline & 40 & 1.36 & $1.2 \cdot 10^{-2}$ & 4.04 & $1.3 \cdot 10^{-1}$ & 25 \\
\hline & 50 & 1.39 & $1.5 \cdot 10^{-2}$ & 2.80 & $1.8 \cdot 10^{-1}$ & 14 \\
\hline \multirow[t]{6}{*}{$\mathrm{C}$} & 0 & 1.29 & $1.1 \cdot 10^{-2}$ & 10.51 & $1.0 \cdot 10^{-1}$ & 25 \\
\hline & 10 & 1.31 & $1.2 \cdot 10^{-2}$ & 8.55 & $1.1 \cdot 10^{-1}$ & 25 \\
\hline & 20 & 1.33 & $1.3 \cdot 10^{-2}$ & 6.82 & $1.1 \cdot 10^{-1}$ & 25 \\
\hline & 30 & 1.36 & $1.4 \cdot 10^{-2}$ & 5.15 & $1.2 \cdot 10^{-1}$ & 25 \\
\hline & 40 & 1.39 & $1.5 \cdot 10^{-2}$ & 3.72 & $1.3 \cdot 10^{-1}$ & 25 \\
\hline & 50 & 1.42 & $1.7 \cdot 10^{-2}$ & 2.54 & $1.6 \cdot 10^{-1}$ & 18 \\
\hline \multirow[t]{6}{*}{$\mathrm{D}$} & 0 & 1.40 & $4.1 \cdot 10^{-2}$ & 8.22 & $1.8 \cdot 10^{-1}$ & 25 \\
\hline & 10 & 1.43 & $4.3 \cdot 10^{-2}$ & 6.59 & $1.8 \cdot 10^{-1}$ & 25 \\
\hline & 20 & 1.46 & $4.7 \cdot 10^{-2}$ & 5.16 & $2.0 \cdot 10^{-1}$ & 24 \\
\hline & 30 & 1.49 & $4.9 \cdot 10^{-2}$ & 3.82 & $2.1 \cdot 10^{-1}$ & 24 \\
\hline & 40 & 1.46 & $3.2 \cdot 10^{-2}$ & 3.34 & $1.4 \cdot 10^{-1}$ & 7 \\
\hline & 50 & 1.57 & $5.1 \cdot 10^{-2}$ & 2.90 & $9.0 \cdot 10^{-2}$ & 12 \\
\hline
\end{tabular}

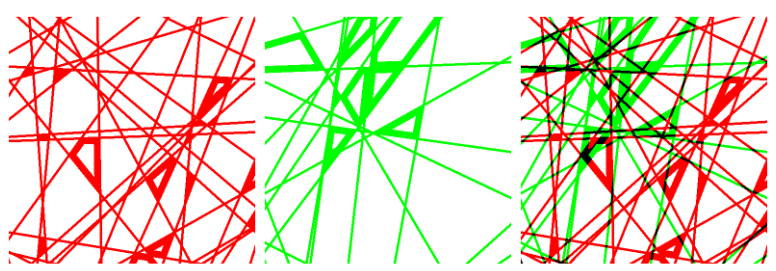

(a)

(b)

(c)

Figure 2: Merging of two adjacent grid layers (a) and (b) to one grid layer (c) 
Table 5: In-plane characteristics of uncompressed and compressed GDLs. BT are binder types A: $b_{r}=6 \mu \mathrm{m}, \mathrm{B}: b_{r}=18 \mu \mathrm{m}, \mathrm{C}: b_{r}=30$ $\mu \mathrm{m}, \mathrm{D}$ : filled pores. CL is the compression level from 0 to $50 \%$.

\begin{tabular}{|c|c|c|c|c|c|c|}
\hline \multirow[t]{2}{*}{ BT } & \multirow{2}{*}{$\begin{array}{l}\text { CL } \\
1 \%\end{array}$} & \multicolumn{2}{|c|}{ Tortuosity $\tau$} & \multicolumn{2}{|c|}{ Permeability / $\mu \mathrm{m}^{2}$} & \multirow{2}{*}{$\begin{array}{l}\text { No. } \\
\text { sim. }\end{array}$} \\
\hline & & mean & $\begin{array}{l}\text { var. } \\
\text { coef. }\end{array}$ & mean & $\begin{array}{l}\text { var. } \\
\text { coef. }\end{array}$ & \\
\hline \multirow[t]{6}{*}{$\mathrm{A}$} & 0 & 1.12 & $4.8 \cdot 10^{-3}$ & 17.63 & $7.9 \cdot 10^{-2}$ & 25 \\
\hline & 10 & 1.12 & $5.1 \cdot 10^{-3}$ & 14.41 & $8.1 \cdot 10^{-2}$ & 25 \\
\hline & 20 & 1.13 & $5.7 \cdot 10^{-3}$ & 11.60 & $8.4 \cdot 10^{-2}$ & 25 \\
\hline & 30 & 1.15 & $6.6 \cdot 10^{-3}$ & 8.91 & $8.5 \cdot 10^{-2}$ & 25 \\
\hline & 40 & 1.17 & $7.9 \cdot 10^{-3}$ & 6.72 & $8.5 \cdot 10^{-2}$ & 25 \\
\hline & 50 & 1.22 & $1.4 \cdot 10^{-2}$ & 4.45 & $9.9 \cdot 10^{-2}$ & 25 \\
\hline \multirow[t]{6}{*}{$\mathrm{B}$} & 0 & 1.11 & $6.3 \cdot 10^{-3}$ & 17.98 & $1.1 \cdot 10^{-1}$ & 25 \\
\hline & 10 & 1.12 & $6.7 \cdot 10^{-3}$ & 14.70 & $1.1 \cdot 10^{-1}$ & 25 \\
\hline & 20 & 1.13 & $7.2 \cdot 10^{-3}$ & 11.85 & $1.1 \cdot 10^{-1}$ & 25 \\
\hline & 30 & 1.15 & $8.3 \cdot 10^{-3}$ & 9.08 & $1.1 \cdot 10^{-1}$ & 25 \\
\hline & 40 & 1.16 & $9.8 \cdot 10^{-3}$ & 6.84 & $1.1 \cdot 10^{-1}$ & 25 \\
\hline & 50 & 1.22 & $1.7 \cdot 10^{-2}$ & 4.45 & $1.3 \cdot 10^{-1}$ & 25 \\
\hline \multirow[t]{6}{*}{$\mathrm{C}$} & 0 & 1.11 & $4.4 \cdot 10^{-3}$ & 17.81 & $9.2 \cdot 10^{-2}$ & 25 \\
\hline & 10 & 1.12 & $4.8 \cdot 10^{-3}$ & 14.56 & $9.4 \cdot 10^{-2}$ & 25 \\
\hline & 20 & 1.13 & $5.4 \cdot 10^{-3}$ & 11.73 & $9.8 \cdot 10^{-2}$ & 25 \\
\hline & 30 & 1.14 & $6.4 \cdot 10^{-3}$ & 9.00 & $1.0 \cdot 10^{-1}$ & 25 \\
\hline & 40 & 1.16 & $7.7 \cdot 10^{-3}$ & 6.79 & $1.0 \cdot 10^{-1}$ & 25 \\
\hline & 50 & 1.22 & $1.3 \cdot 10^{-2}$ & 4.39 & $1.3 \cdot 10^{-1}$ & 25 \\
\hline \multirow[t]{6}{*}{$\mathrm{D}$} & 0 & 1.12 & $4.2 \cdot 10^{-3}$ & 16.93 & $9.9 \cdot 10^{-2}$ & 25 \\
\hline & 10 & 1.12 & $4.4 \cdot 10^{-3}$ & 13.82 & $1.0 \cdot 10^{-1}$ & 25 \\
\hline & 20 & 1.13 & $4.8 \cdot 10^{-3}$ & 11.10 & $1.1 \cdot 10^{-1}$ & 25 \\
\hline & 30 & 1.15 & $1.3 \cdot 10^{-2}$ & 8.49 & $1.1 \cdot 10^{-1}$ & 25 \\
\hline & 40 & 1.17 & $6.6 \cdot 10^{-3}$ & 6.37 & $1.1 \cdot 10^{-1}$ & 25 \\
\hline & 50 & 1.23 & $1.4 \cdot 10^{-2}$ & 4.13 & $1.5 \cdot 10^{-1}$ & 25 \\
\hline
\end{tabular}

Table 7: Deviation of the through-plane simulations from the KozenyCarman trend (eq. (5)), average values over up to 25 simulations each. Compression level CL from 10 to $50 \%$ for binder types A, B, C, D.

\begin{tabular}{cccccc} 
CL & $10 \%$ & $20 \%$ & $30 \%$ & $40 \%$ & $50 \%$ \\
\hline A & $2.5 \%$ & $5.9 \%$ & $9.6 \%$ & $14.8 \%$ & $36.3 \%$ \\
B & $2.8 \%$ & $6.5 \%$ & $10.5 \%$ & $16.1 \%$ & $37.2 \%$ \\
C & $3.2 \%$ & $7.1 \%$ & $11.4 \%$ & $17.1 \%$ & $39.1 \%$ \\
D & $4.4 \%$ & $9.5 \%$ & $15.2 \%$ & $16.1 \%$ & $17.2 \%$
\end{tabular}
$\begin{array}{llllll}4.4 \% & 9.5 \% & 15.2 \% & 16.1 \% & 17.2 \%\end{array}$

Table 8: Deviation of the in-plane simulations from the KozenyCarman trend (eq. (5)), average values over 25 simulations each. Compression level CL from 10 to $50 \%$ for binder types A, B, C,

\begin{tabular}{cccccc}
$\mathrm{CL}$ & $10 \%$ & $20 \%$ & $30 \%$ & $40 \%$ & $50 \%$ \\
\hline $\mathrm{A}$ & $3.4 \%$ & $7.2 \%$ & $10.7 \%$ & $13.5 \%$ & $35.4 \%$ \\
$\mathrm{~B}$ & $3.4 \%$ & $7.1 \%$ & $10.7 \%$ & $13.7 \%$ & $36.0 \%$ \\
$\mathrm{C}$ & $3.4 \%$ & $7.2 \%$ & $10.7 \%$ & $13.6 \%$ & $36.6 \%$ \\
$\mathrm{D}$ & $3.7 \%$ & $7.6 \%$ & $11.3 \%$ & $14.6 \%$ & $37.5 \%$ \\
& \\
Boundaries & Level of compression \\
between \\
fiber layers
\end{tabular}

Figure 3: Compression of the virtual geometry in steps of $10 \%$

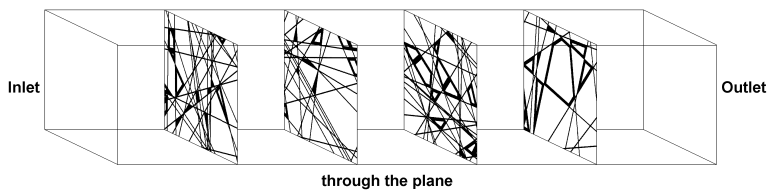

Figure 4: Simulation frame of through-plane simulations numbers of simulations. Compression level CL from 0 to $30 \%$ for binder type $\mathrm{D}$.

\begin{tabular}{cllllll} 
CL & & $\mathrm{N}=5$ & $\mathrm{~N}=10$ & $\mathrm{~N}=15$ & $\mathrm{~N}=20$ & $\mathrm{~N}=25$ \\
\hline 0 & mean & 7.73 & 8.15 & 8.17 & 8.44 & 8.22 \\
& var. coef. & 0.13 & 0.22 & 0.19 & 0.18 & 0.18 \\
10 & mean & 6.17 & 6.52 & 6.55 & 6.77 & 6.59 \\
& var. coef. & 0.13 & 0.23 & 0.20 & 0.18 & 0.18 \\
20 & mean & 4.82 & 5.11 & 5.14 & 5.28 & 5.16 \\
& var. coef. & 0.15 & 0.24 & 0.21 & 0.20 & 0.20 \\
30 & mean & 3.56 & 3.79 & 3.81 & 3.92 & 3.82 \\
& var. coef. & 0.16 & 0.25 & 0.23 & 0.21 & 0.21
\end{tabular}

${ }^{a} \mathrm{~N}=24$ for 20 and $30 \%$ compression

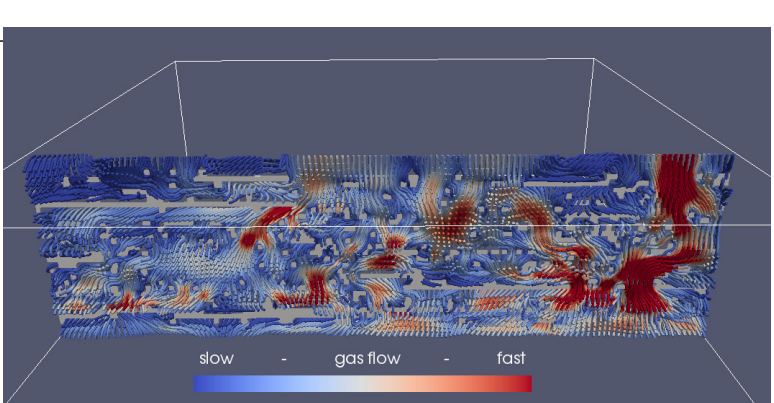

Figure 5: Path lines of a transport simulation through a type B geometry. Gas is flowing upwards in $\mathrm{x}$ direction. 


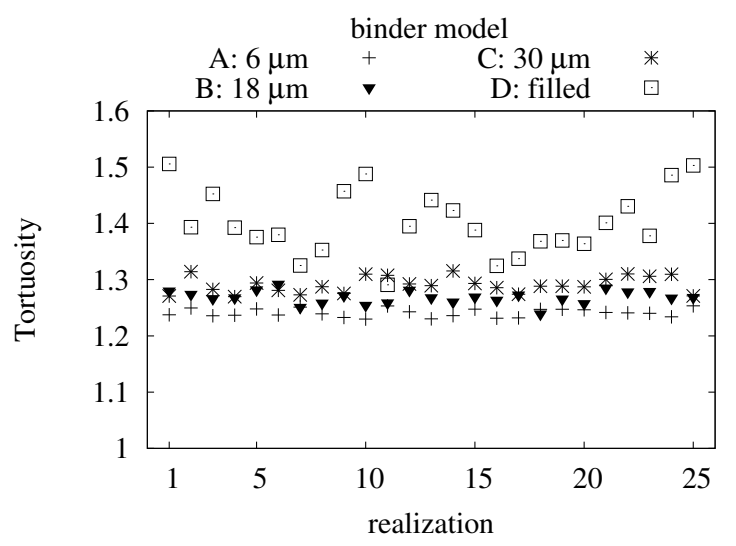

Figure 6: Tortuosity in through-plane simulations, uncompressed

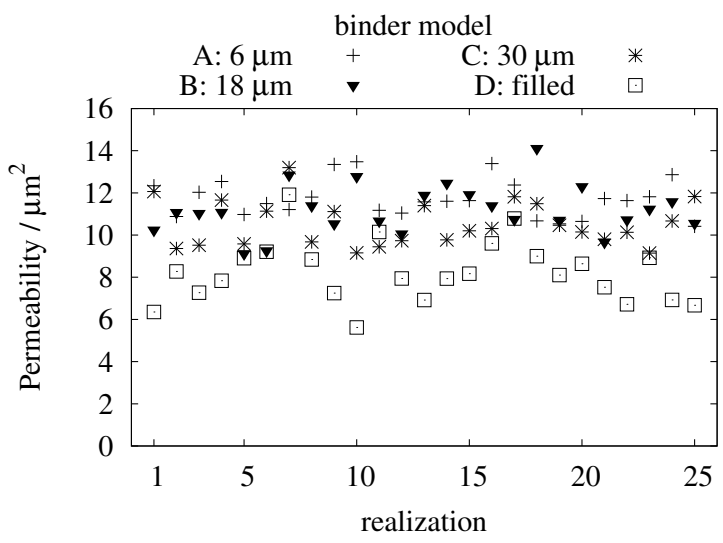

Figure 7: Permeability in through-plane simulations, uncompressed

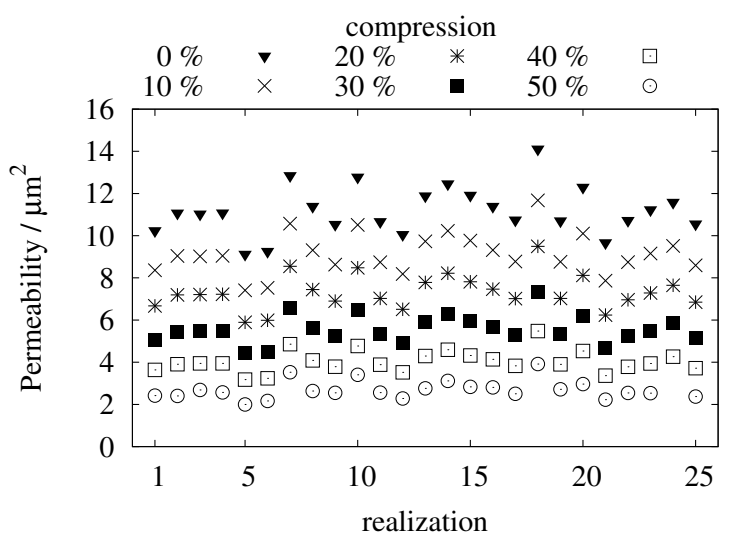

Figure 8: Through-plane permeability in compressed GDLs, binder model $\mathrm{B}, b_{r}=18 \mu \mathrm{m}$

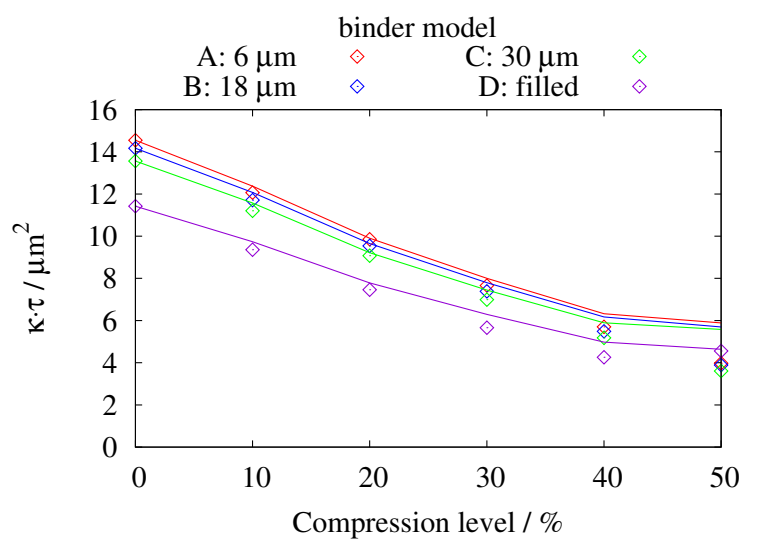

Figure 9: Simulated through-plane permeability (symbols) and Kozeny-Carman trend (lines) in compressed GDLs

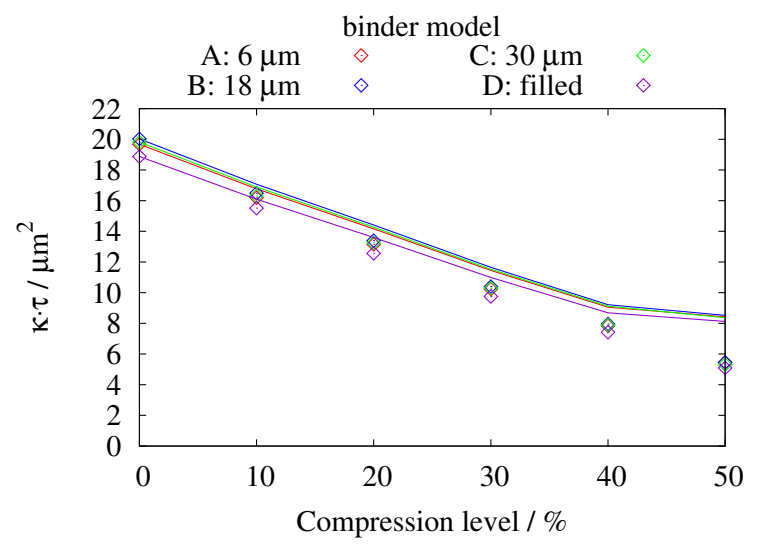

Figure 10: Simulated in-plane permeability (symbols) and KozenyCarman trend (lines) in compressed GDLs 